\title{
Integron in Gram negative bacteria: Classes and fitness cost
}

\author{
Aisha M AlAmri \\ Assistant Professor, Medical Microbiology, Clinical Lab Sciences Department, College of Applied Medical Sciences, \\ Imam Abdulrahman Bin Faisal University, Dammam, Saudi Arabia
}

Bacteria intrinsically harbor methods of gene exchange that are distinct from those in eukaryotes, but subject to natural selection laws. Dissemination of antimicrobial resistance is one of the major consequences of gene exchange ability. Bacterial genomes have the ability to undergo evolutionary changes within specified time frame resulting in an exceptional diversity, especially, under strong selective pressure. One of the most important elements of antimicrobial resistance are integrons which serve as a platform for gene cassettes integration and expression.

\section{Access this article online}

Website:

http://nepjol.info/index.php/AJMS

DOI: $10.3126 /$ ajms.v8i6.18339

E-ISSN: 2091-0576

P-ISSN: 2467-9100

Key words: Integron, Integrase, Fitness cost, Gene cassette

\section{INTRODUCTION}

Integrons were characterized in the late 1980s as a major contributor to the dissemination of antibiotic resistant determinants among Gram-negative bacteria. ${ }^{1}$ Though each has unique features, chromosomal and mobile integrons exist with the latter, as the name implies, carried on mobile elements such as transposons and plasmids and largely associated with antibiotic resistance. The general structure of an integron includes an integrase gene, gene cassettes and a recombination site as well as two conserved regions, one at each end of the integron structure: the 5' and 3' conserved sequences (CS). ${ }^{2}$

Integrons are classified according to their integrase (intI) gene sequence. Integrase is a tyrosine recombinase enzyme however; it has been found that integrase differs from the other tyrosine recombinase family members in a certain motif that is unique for integrase and is essential for its activity. ${ }^{3}$ It has recently been shown that stress responses such as the SOS response may lead to integrase overexpression, thus increasing the likelihood of gene cassette rearrangement in integrons. ${ }^{4}$

Gene cassettes are usually promoter-less and consisting of an open reading frame and an adjacent recombination site known as 59 be or attC, specifically recognized by the integrase enzyme. Multiple gene cassettes assembled into an array could be included in a single integron structure; however, integrons with no gene cassettes have also been observed. The majority of gene cassettes identified are associated with antibiotic resistance. An integron general promoter (Pc)located within the sequence of the intI gene directs the expression of all the gene cassettes. Additionally, a second promoter may also exist in some integrons. The strength of expression between the gene cassettes is variable depending on how close each gene cassette is to the promoter region meaning that proximal gene cassettes are expressed more strongly than distal ones. This is believed to be due to transcription attenuation with the transcript span. ${ }^{5}$ Other factors that could affect the level of expression include the presence of internal promoters. The 59 be are of variable length between 57-141 bp with 25 bp terminal inverted repeats (Figure 1). This article aims to go through the common classes of integrons, their structure, medical importance and fitness cost.

\section{Class 1 integron}

In Class 1 integrons, the 5'-CS includes the intI1 gene whereas the 3'-CS contains the qacE (encoding quaternary ammonium compounds resistance)and sull (encoding sulfonamide resistance)genes in addition to an open reading frame (ORF)with unknown function. ${ }^{6}$

An atypical 3'CS is also observed in some cases, such as the presence of another ORF insertion of qacE as 


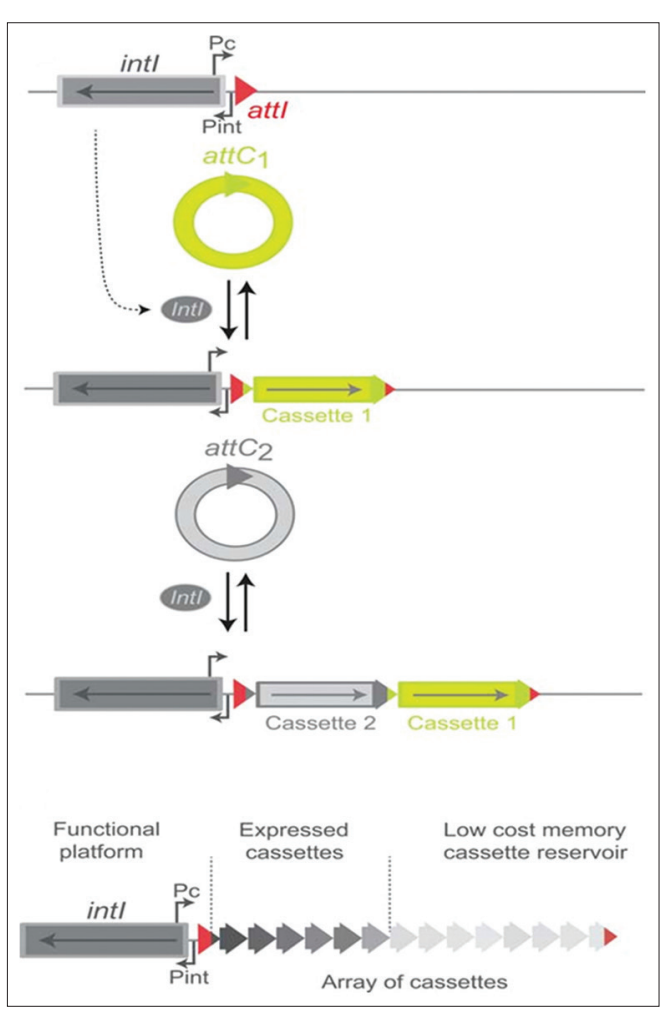

Figure 1: General structure of an integron and the gene cassette (GC) integration/excision mechanism. The integrase enzyme, encoded by intl, catalyzes the insertion and the excision of the gene cassettes. Adapted from Escudero et al. ${ }^{2}$

a gene cassette or deletion of some regions. ${ }^{7}$ Integrons themselves are not mobile but they are normally carried and mobilized by transposons. It has been proposed that some integrons are defective transposons, which suggests an evolutionary history of integrons that lost their transposition functions. ${ }^{8}$

\section{Class 2 integron}

Class 2 integrons typically have a defective, nonfunctional integrase encoded by a gene that shares $46 \%$ homology with intI1 commonly found to be associated with the $\operatorname{Tn} 7$ transposon family and its derivatives (Tn1825, Tn1826 and Tn4132) with its Promoter (Pc) and recombination site attI 2 inserted within the transposons. ${ }^{9}$ Classically, class 2 integrons contains an array of gene cassettes, including dihydrofolate reductase $(d f r A 1)$, streptothricin acetyltransferase (sat1), and aminoglycoside adenyltransferase (adA1), confering resistance to trimethoprim, streptothricin and streptomycin/spectinomycin, respectively. ${ }^{10}$ Additionally, class 2 integrons share some gene cassettes with class 1 integrons, including $d f r A 1$, sat1 and aad $A 1 .{ }^{11}$

Class 2 integrons is generally less prevalent than class 1 integron, and have so far been reported in a range of Gramnegative organisms such as Acinetobacter, Enterobacteriaceae, Salmonella and Psuedomonas. ${ }^{12}$
It has been shown in E. coli, that intI2 is capable of specific excision and integration of gene cassettes precisely into attI2, for instance, erythromycin esterase gene (ereA) was found to be associated with class 2 integron including its own promoter along with an insertion sequence element (IS1)upstream of the intI2 gene. ${ }^{13}$ In Acinetobacter baumannii, a novel rearrangement of a class 2 integron with new cassettes in the variable region were identified and found to be different antibiotic resistance genes (sat2, aadB and catB2) inserted upstream of the 3 conventional antibiotic resistance genes of $\operatorname{Tn} 7$ class 2 integron. ${ }^{14}$

\section{Class 3 integron}

Class 3 integron shares comparable functions to IntI1 and was found to be associated with soil and freshwater Proteobacteria group. This class was first isolated from Serratia marcescens strains acquiring bla ${ }_{\mathrm{IMP}}$ in Japan in 1993 followed by the identification of Klebsiella pneumoniae harbouring bla ${ }_{\mathrm{GES}-1}$ gene. ${ }^{15}$ This class is less frequent than class 1 and 2 and so far has been identified in a range of Gram Negative species such as Acinetobacter spp., Citrobacter freundii, Alcaligenes Escherichia coli, Klebsiella pneumoniae, Pseudomonas aeruginosa, Serratia marcescens and Salmonella spp. ${ }^{16}$

Class 3 integrons in Gram-negative bacteria was also isolated from hospital sewage containing Acinetobacter spp, Aeromonas spp and Citrobacter freundii with oxacillinase gene cassette as well as cassettes encoding aminoglycoside and $\beta$-lactam resistance implicated. ${ }^{17}$

\section{Epidemiology of integrons}

Integrons are ubiquitous in nature. Since their discovery, they gained considerable attention, mainly because of their association with the dissemination of antibiotic resistance. The widespread nature of integrons and their stability triggered lots of investigations about their evolution and distribution in diverse environments. ${ }^{18}$

One study aimed to assess the prevalence of class 1 integrons among bacterial isolates from European hospitals in nine countries. The authors demonstrated that $43 \%$ of 163 unrelated Gram-negative isolates were integron positive, carrying resistance to aminoglycosides, fluoroquinolones and $\beta$-lactams including the third generation cephalosporins and monobactams. ${ }^{19}$ Schmitz et al assessed 278 strains representing 11 different Gram-negative species isolated from blood samples for the presence of integrons. ${ }^{20}$ Their work showed that $13 \%$ of the isolates (belonging to six species)carried integron structures. The increased prevalence of integrons, and particularly of those with several gene cassettes over time is also evident in the clinical settings were the selective pressure is high. Another study by Schmitz et al targeted the prevalence of integrons among enterobacterial

Asian Journal of Medical Sciences | Nov-Dec 2017 | Vol 8 | Issue 6 
blood-culture isolates, demonstrating that their prevalence elevated from $4.7 \%$ in 1993 to $17.4 \%$ in $1999 .{ }^{21}$ Studies targeting the prevalence of integrons in China indicated a level of 52\% among E. colic linical isolates. ${ }^{22}$ Another Chinese study conducted 6 years later found that nearly $85 \%$ of E. coli clinical isolates were integron positive. ${ }^{22}$ In the USA, a study estimated the prevalence of class 1 integrons among E. coli isolates of human and animal origin and showed that among 274 isolates, $16 \%$ were found to carry class 1 integrons. ${ }^{23}$

Several studies have suggested that mobile integrons carrying antibiotic resistance gene cassettes are widely spread among bacterial genomes due to the direct or indirect selective pressure of antibiotic use in clinical and environmental settings. ${ }^{24,25}$ Certainly, even in the absence of antibiotic selection, factors such as heavy metals and the presence of quaternary ammonium compounds could also select for the dissemination of mobile integrons. Studying the faecal carriage of E. coli in animals, Skurnik et al noticed that the close proximity of humans to animals increases the prevalence of integrons in the animals' bacterial flora..$^{25}$

When Stokes et al examined bacteria isolated from soil and sediments where no obvious antibiotic selection existed, class 1 integrons was identified from 4 different bacterial species not known to be present in humans. ${ }^{26}$ None of the 4 integrons isolated had antibiotic resistance gene cassettes in them. The study suggested that evolution and dissemination of integrons may have begun before the antibiotic era. Recently Khosravi et al screened 93 P. aeuroginosa strains isolated from blood and wound, for the presence of class 1 and 2 integron. ${ }^{27}$ Their result revealed the presence of class 1 integron in all of blood isolates and in $95.38 \%$ of wound isolates but no class 2 integron was detected.

In China, class 1 and 2 integrons were also identified in $45.8 \%(54 / 118)$ and $19.5 \%(23 / 118)$ of Pseudomonas aeruginosa isolates, respectively. ${ }^{10}$

In 2016, Kheiri et al, tested 200 E. coli isolates, 136 isolates were multi drug resistance (MDR)collected from chicken, human, cattle and sheep. Class 1 was detected in 38\% of human isolates relative to $72 \%$ of animal isolates. ${ }^{28}$ On the other hand, class 2 was found in $8 \%$ and $30 \%$ of human and animal isolates respectively. ${ }^{28}$

In another study performed on 164 E. coli isolates, class 1 and class 2 integrons and class 3 were found in $78.26 \%$, $76.81 \%$ and $26.09 \%$ MDR isolates, respectively. ${ }^{29}$

A surveillance study investigated the occurrence of class 3 integron among 587 Gram negative isolates reported the prevalence of class 3 integron to be $0.7 \% .^{30}$

\section{Fitness cost of integrons}

The evolution of antibiotic resistance imposes a fitness cost, demonstrated as reduced growth rate of the resistant pathogen. fitness cost plays a major role in shaping the level of resistance particularly when the selective pressure is high. Studies have shown that the cost of resistance is highly variable among different strains carrying a wide range of resistant determinant. Since integrons serve as a platform for resistant gene cassettes then the fitness cost of the entire integron structure is dependent on that of every gene cassette carried. Starikova et al demonstrated that the newly acquired class-1 integrons from Salmonella enterica serovar Typhimurium and Acinetobacter baumannii significantly reduced the host fitness in recipient strain of Acinetobacter baylyi. ${ }^{31}$ Interestingly, fitness was restored when insertional inactivation of the acquired integron intI 1 was applied.

Similar observation was made by Klus et al who noticed elevated fitness cost in the strain studied (Acinetobacter baylyz)suggesting its genetic instability. ${ }^{32}$

\section{CONCLUSION}

Horizontal gene transfer is one of the most vital bacterial weapons. Integrons are a professional system by which acquiring and rearrangement of gene cassettes take place. The problem is worse when these integrons are located on a mobile element such as plasmids and transposons. Wide range of Gram negative bacteria where found to harbor these structures with most of the gene cassettes discovered are known to express antibiotics resistance and this has a major clinical implication. Fitness cost of the integron structure is yet another clinically relevant concern especially in this era where Multiple drug resistant isolates are causing epidemics threatening the limited antimicrobial options.

\section{REFERENCES}

1. Stokes HW and Hall RM. A novel family of potentially mobile DNA elements encoding site-specific gene-integration functions: integrons. Mol Microbiol1989; 3(12):1669-1683.

2. Escudero JA, Loot C, Nivina A and Mazel D. The Integron: Adaptation On Demand. Microbiol Spectr 2015; 3(2): MDNA30019-2014.

3. Messier $\mathrm{N}$ and Roy $\mathrm{PH}$. Integron integrases possess a unique additional domain necessary for activity. J Bacteriol 2001;183(22): 6699-6706.

4. Guerin E, Cambray G, Da Re S, Mazel D and Ploy MC. The SOS response controls integron recombination. Science 2009; 324(5930):1034.

5. Collis $\mathrm{CM}$ and Hall RM. Expression of antibiotic resistance genes in the integrated cassettes of integrons. Antimicrob Agents Chemother 1995; 39(1): 155-162.

6. Paulsen IT, Littlejohn TG, Radstrom P, Sundstrom L, Skold O, Swedberg G, et al. The 3' conserved segment of integrons contains a gene associated with multidrug resistance to 
antiseptics and disinfectants. Antimicrob Agents Chemother 1993; 37(4): 761-768.

7. Partridge SR, Recchia GD, Stokes HW and Hall RM. Family of class 1 integrons related to In4 from Tn1696. Antimicrob Agents Chemother 2001; 45(11): 3014-3020.

8. Brown HJ, Stokes HW and Hall RM. The integrons In0, In2, and In5 are defective transposon derivatives. J Bacteriol 1996; 178(15): 4429-4437.

9. Jove T, Da Re S, Tabesse A, Gassama-Sow A and Ploy MC Gene Expression in Class 2 Integrons Is SOS-Independent and Involves Two Pc Promoters. Front Microbiol 2017; 8: 1499.

10. Xu Z, Li L, Shirtliff ME, Alam MJ, Yamasaki S and Shi L. Occurrence and characteristics of class 1 and 2 integrons in Pseudomonas aeruginosa isolates from patients in southern China. J Clin Microbiol 2009; 47(1): 230-234.

11. Hansson K, Sundstrom, L, Pelletier A and Roy PH. Intl2 integron integrase in Tn7. J Bacteriol 2002; 184(6): 1712-1721.

12. Macedo-Vinas M, Cordeiro NF, Bado I, Herrera-Leon S, Vola M, Robino L, et al. Surveillance of antibiotic resistance evolution and detection of class 1 and 2 integrons in human isolates of multi-resistant Salmonella Typhimurium obtained in Uruguay between 1976 and 2000. Int J Infect Dis 2009; 13(3): 342-348.

13. Biskri L and Mazel $D$. Erythromycin esterase gene ere(A)is located in a functional gene cassette in an unusual class 2 integron. Antimicrob Agents Chemother 2003; 47(10): 3326-3331.

14. Ramirez MS, Quiroga $C$ and Centron D. Novel rearrangement of a class 2 integron in two non-epidemiologically related isolates of Acinetobacter baumannii. Antimicrob Agents Chemother 2005; 49(12): 5179-5181.

15. Correia M, Boavida F Grosso F, Salgado MJ, Lito LM, Cristino JM, et al. Molecular characterization of a new class 3 integron in Klebsiella pneumoniae. Antimicrob Agents Chemother 2003; 47(9): 2838-2843.

16. Rowe-Magnus D, GueroutA, Ploncard AM, Dychinco P, Davies BJ and Mazel D. The evolutionary history of chromosomal superintegrons provides an ancestry for multiresistant integrons. Proc Natl Acad Sci U S A 2001; 98(2): 652-657.

17. Simo Tchuinte PL, Stalder T, Venditti S, Ngandjio A, Dagot C, Ploy $\mathrm{MC}$, et al. Characterisation of class 3 integrons with oxacillinase gene cassettes in hospital sewage and sludge samples from France and Luxembourg. Int J Antimicrob Agents 2016; 48(4): 431-434.

18. Leverstein-van Hall MAM, Blok HET, Donders AR, Paauw A, Fluit $A C$ and Verhoef J. Multidrug resistance among Enterobacteriaceae is strongly associated with the presence of integrons and is independent of species or isolate origin. $\mathrm{J}$ Infect Dis 2003; 187(2): 251-259.

19. Martinez-Freijo P, Fluit AC, Schmitz FJ, Grek VS, Verhoef J and Jones ME. Class I integrons in Gram-negative isolates from different European hospitals and association with decreased susceptibility to multiple antibiotic compounds. J Antimicrob Chemother 1998; 42(6): 689-696.

20. Schmitz FJ, Martinez-Freijo P, Theis S, Fluit AC, Verhoef J,
Heinz HP, et al. Class I integrons: prevalence and impact on antibiotic susceptibility in 278 consecutive unrelated Gramnegative blood isolates. Clin Microbiol Infect 1999; 5(8): 496-498.

21. Schmitz FJ, Hafner D, Geisel R, Follmann P, Kirschke C, Verhoef $\mathrm{J}$, et al. Increased prevalence of class I integrons in Escherichia coli, Klebsiella species, and Enterobacter species isolates over a 7-year period in a German university hospital. J Clin Microbiol 2001; 39(10): 3724-3746.

22. Chang CY, Chang LL, Chang YH, Lee TM and Chang SF. Characterisation of drug resistance gene cassettes associated with class 1 integrons in clinical isolates of Escherichia coli from Taiwan, ROC. J Med Microbiol 2000; 49(12): 1097-1102.

23. Singh R, Schroeder CM, Meng J, White DG, McDermott PF, Wagner DD, et al. Identification of antimicrobial resistance and class 1 integrons in Shiga toxin-producing Escherichia coli recovered from humans and food animals. J Antimicrob Chemother 2005; 56(1): 216-219.

24. Skurnik D, Le Menac'h A, Zurakowski D, Mazel D, Courvalin P, Denamur E, et al. Integron-associated antibiotic resistance and phylogenetic grouping of Escherichia coli isolates from healthy subjects free of recent antibiotic exposure. Antimicrob Agents Chemother 2005; 49(7): 3062-3065.

25. Daikos GL, Kosmidis C, Tassios PT, Petrikkos G, Vasilakopoulou A, Psychogiou M, et al. Enterobacteriaceae bloodstream infections: presence of integrons, risk factors, and outcome. Antimicrob Agents Chemother 2007; 51(7): 2366-2272.

26. Stokes HW, Nesbo CL, Holley M, Bahl MI, Gillings MR and Boucher Y. Class 1 integrons potentially predating the association with tn402-like transposition genes are present in a sediment microbial community. J Bacteriol 2006; 188(16): 5722-5730.

27. Khosravi $A D$, Motahar $M$ and Abbasi Montazeri $E$. The frequency of class 1 and 2 integrons in Pseudomonas aeruginosa strains isolated from burn patients in a burn center of Ahvaz, Iran. PLoS One 2017; 12(8): e0183061.

28. Kheiri $\mathrm{R}$ and Akhtari L. Antimicrobial resistance and integron gene cassette arrays in commensal Escherichia coli from human and animal sources in IRI. Gut Pathog 2016; 8(1): 40.

29. Kargar M, Mohammadalipour Z, Doosti Z, Lorzadeh AS and Japoni-Nejad A. High Prevalence of Class 1 to 3 Integrons Among Multidrug-Resistant Diarrheagenic Escherichia coli in Southwest of Iran. Osong Public Health Res Perspect 2014; 5(4): 193-198.

30. Shibata N, Doi Y, Yamane K, Yagi T, Kurokawa H, Shibayama K, et al. PCR typing of genetic determinants for metallo-betalactamases and integrases carried by gram-negative bacteria isolated in Japan, with focus on the class 3 integron. J Clin Microbiol 2003; 41(12): 5407-5413.

31. Starikova I, Harms K, Haugen P, Lunde TT, Primicerio R, Samuelsen $O$, et al. A trade-off between the fitness cost of functional integrases and long-term stability of integrons. PLoS Pathog 2012; 8(11): e1003043.

32. Harms K, Starikova I and Johnsen PJ. Costly Class-1 integrons and the domestication of the the functional integrase. Mob Genet Elements 2013; 3(2): e24774.

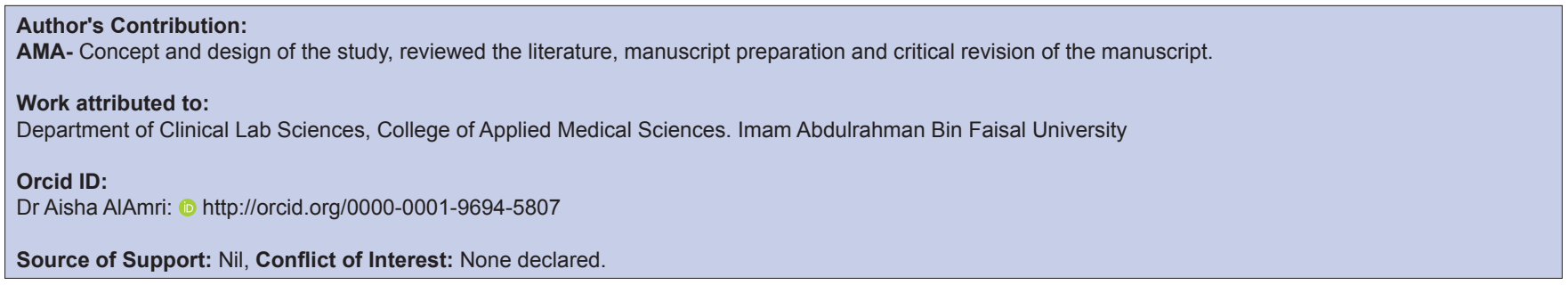

